\title{
Non-local Problem for the Loaded Integral-differential Equation in Double-connected Domain
}

\author{
ABDULLAYEV O. $\mathrm{Kh}^{*}$ \\ National University of Uzbekistan, Tahskent.
}

Received 24 October 2015; Accepted 15 January 2016

\begin{abstract}
In this work an existence and uniqueness of solution of the non-local boundary value problem for the loaded elliptic-hyperbolic type equation with integral-differential operations in double-connected domain have been investigated. The uniqueness of solution is proved by the method of integral energy using an extremum principle for the mixed type equations, and the existence is proved by the method of integral equations.
\end{abstract}

\section{AMS Subject Classifications: 35M10 \\ Chinese Library Classifications: 0175.28}

Key Words: Loaded equation; elliptic-hyperbolic type; integral-differential operators; doubleconnected domain; an extremum principle; existence of solution; uniqueness of solution; method of integral energy; integral equations.

\section{Introduction and formulation of a problem}

We note that with intensive research on problem of optimal control of the agroeconomical system, regulating the label of ground waters and soil moisture, it has become necessary to investigate a new class of equations called "LOADED EQUATIONS". For the first time it was given the most general definition of a Loaded equations and various Loaded equations are classified in detail by A.M.Nakhushev [1]. After this work very interesting results on the theory of boundary value problems for the loaded equations of parabolic, parabolic-hyperbolic and elliptic-hyperbolic types were published, for example, see [2-4] and [5]. In this direction, we studied some local and non-local problems for the loaded second and third order elliptic-hyperbolic type equations in double-connected domains

${ }^{*}$ Corresponding author. Email address: obidjon.mth@gmail . com (Abdullayev O. Kh) 
(see [6-9]). Notice that non-local problems for the loaded integral-differential elliptichyperbolic type equations in double-connected domains have not been investigated. In the given paper, for the equation:

$$
u_{x x}+\operatorname{sgn} y u_{y y}+\frac{1-\operatorname{sgn} y}{2} \sum_{k=1}^{n} R_{k}(x, 0)=0
$$

with operators [10]:

$$
\begin{aligned}
& R_{k}(x, y)=R_{k}\left(\frac{\xi+\eta}{2}, \frac{\xi-\eta}{2}\right)= \begin{cases}p_{k}(\xi) D_{\xi 1}^{\alpha_{k}} u\left(\frac{\xi+\eta}{2}, \frac{\xi-\eta}{2}\right), & \text { at } q \leq x \leq 1, \\
r_{k}(\eta) D_{-1 \eta}^{\beta_{k}} u\left(\frac{\xi+\eta}{2}, \frac{\xi-\eta}{2}\right), & \text { at }-1 \leq x \leq-q,\end{cases} \\
& D_{a x}^{\alpha_{k}} f(x)=\frac{1}{\Gamma\left(-\alpha_{k}\right)} \int_{a}^{x}(x-t)^{-1-\alpha_{k}} f(t) \mathrm{d} t, \quad-1<\alpha_{k}<0, \\
& D_{x a}^{\beta_{k}} f(x)=\frac{1}{\Gamma\left(-\alpha_{k}\right)} \int_{x}^{a}(t-x)^{-1-\beta_{k}} f(t) \mathrm{d} t, \quad-1<\beta_{k}<0,
\end{aligned}
$$

we will investigate the uniqueness and the existence of solution of the non-local problem. Let's, $\Omega$, be double connected domain, bounded with two lines:

$$
\sigma_{1}: x^{2}+y^{2}=1 ; \sigma_{2}: x^{2}+y^{2}=q^{2}, y>0,
$$

and characteristics:

$$
A_{j} C_{1}: x+(-1)^{j} y=(-1)^{j+1} ; B_{j} C_{2}: x+(-1)^{j} y=(-1)^{j+1} \cdot q ;(0<q<1),(j=1,2)
$$

of the Eq. (1.1) at $y<0$, where $x+y=\xi, x-y=\eta ; A_{1}(1 ; 0), A_{2}(-1 ; 0), B_{1}(q ; 0), B_{2}(-q ; 0)$, $C_{1}(0 ;-1), C_{2}(0 ;-q)$.

Introduce designations: $\theta_{1}(x)=\frac{x+1}{2}+i \cdot \frac{x-1}{2}, \theta_{2}(x)=\frac{x-1}{2}-i \cdot \frac{x+1}{2},\left(i^{2}=-1\right)$;

$$
\begin{aligned}
& \Omega_{0}=\Omega \cap(y>0), \quad \Delta_{1}=\Omega \cap(x+y>q) \cap(y<0), \quad \Delta_{2}=\Omega \cap(y-x>q) \cap(y<0) ; \\
& D_{1}=\Omega \cap(-q<x+y<q) \cap(x>0), \quad D_{2}=\Omega \cap(-q<y-x<q) \cap(x<0) ; \\
& D_{3}=\Omega \cap(-1<x+y<-q) \cap(-1<y-x<-q), \quad D_{0}=\Omega_{0} \cup \Delta_{1} \cup \Delta_{2} ; \\
& I_{2+j}=\left\{x: 0<(-1)^{j-1} x<q\right\}, \quad I_{j}=\left\{x: \frac{q+1}{2}<(-1)^{j-1} x<1\right\}, \quad(j=1,2) .
\end{aligned}
$$

In the domain $\Omega$ the following problem is investigated.

Problem I. To find a function $u(x, y)$ satisfies the following properties:

1) $u(x, y) \in C(\bar{\Omega})$;

2) $u(x, y)$ is a regular solution of the Eq. (1.1) in the domain of $\Omega \backslash(y-x= \pm q) \backslash(x+y=$ $\pm q)$, besides, $u_{y} \in C\left(A_{1} B_{1} \cup A_{2} B_{2}\right)$ and $u_{y}(x, 0)$ can tend to infinity an order of less unit at $x \rightarrow \pm q$, and finite at $x \rightarrow \pm 1$; 
3) on the line of changing type satisfy gluing condition:

$$
u_{y}(x,-0)=u_{y}(x,+0), \quad(x, 0) \in A_{1} B_{1} \cup A_{2} B_{2} ;
$$

4) $u(x, y)$ satisfies boundary conditions:

$$
\begin{aligned}
& \left.u(x, y)\right|_{\sigma_{j}}=\varphi_{j}(x, y), \quad(x, y) \in \overline{\sigma_{j}} ; \\
& \left.u(x, y)\right|_{B_{j} c_{2}}=g_{j}(x), \quad x \in \overline{I_{2+j}} ; \\
& \frac{\mathrm{d}}{\mathrm{d} x} u\left(\theta_{1}(x)\right)=a_{1}(x) u_{y}(x, 0)+b_{1}(x) u_{x}(x, 0)+c_{1}(x) u(x, 0)+d_{1}(x), \quad x \in(q, 1) ; \\
& \frac{\mathrm{d}}{\mathrm{d} x} u\left(\theta_{2}(x)\right)=a_{2}(x) u_{y}(x, 0)+b_{2}(x) u_{x}(x, 0)+c_{2}(x) u(x, 0)+d_{2}(x), \quad x \in(-1,-q) ;
\end{aligned}
$$

where $\varphi_{j}(x, y), g_{j}(x), a_{j}(x), b_{j}(x), c_{j}(x)$, are given functions, such that: $g_{1}(0)=g_{2}(0)$, $g_{2}(-q)=\varphi_{2}(-q, 0), g_{1}(q)=\varphi_{2}(q, 0),(j=1,2)$.

\section{The uniqueness of solution of the Problem I}

Known that the Eq. (1.1) at $y \leq 0$ on the characteristics coordinate $\xi=x+y$ and $\eta=x-y$ has a form:

$$
u_{\xi \eta}=\frac{1}{4} \sum_{k=1}^{n} R_{k}(\xi, 0) .
$$

Notice, that solution the Cauchy problem for the Eq. (1.1) in the domain of $\Delta_{1}$ with conditions $u(x, 0)=\tau_{1}(x), x \in \overline{A_{1} B_{1}}$ and $u_{y}(x, 0)=v_{1}(x), x \in A_{1} B_{1}$, with the account Riemannt function $R\left(\xi, \eta ; \xi_{0}, \eta_{0}\right)=1$ of the Eq. (2.1), looks like:

$$
u(x, y)=\frac{\tau_{1}(x+y)+\tau_{1}(x-y)}{2}-\frac{1}{2} \int_{x+y}^{x-y} v_{1}(t) \mathrm{d} t+\frac{1}{4} \int_{x+y}^{x-y} \mathrm{~d} \xi \int_{\xi}^{x-y} \sum_{k=1}^{n} p_{k}(\xi) D_{\xi 1}^{\alpha_{k}} \tau_{1}(\xi) \mathrm{d} \eta
$$

From here, considering

$$
u\left[\theta_{1}(x)\right]=\frac{\tau_{1}(x)+\tau_{1}(1)}{2}-\frac{1}{2} \int_{x}^{1} v_{1}(t) \mathrm{d} t+\frac{1}{4} \sum_{k=1}^{n} \int_{x}^{1}(1-\xi) p_{k}(\xi) D_{\xi 1}^{\alpha_{k}} \tau_{1}(\xi) \mathrm{d} \xi
$$

and by virtue (1.7) we will get:

$$
\begin{gathered}
\frac{1}{2} \tau_{1}^{\prime}(x)+\frac{1}{2} v_{1}(x)+\frac{x-1}{4} \sum_{k=1}^{n} p_{k}(x) D_{x 1}^{\alpha_{k}} \tau_{1}(x)=a_{1}(x) v_{1}(x)+b_{1}(x) \tau_{1}^{\prime}(x)+c_{1}(x) \tau_{1}(x)+d_{1}(x) \\
\left(2 a_{1}(x)-1\right) v_{1}(x)=\left(1-2 b_{1}(x)\right) \tau_{1}^{\prime}(x)-\frac{1-x}{2} \sum_{k=1}^{n} p_{k}(x) D_{x 1}^{\alpha_{k}} \tau_{1}(x) \\
-2 c_{1}(x) \tau_{1}(x)-2 d_{1}(x) .
\end{gathered}
$$


Precisely also, from the solution

$$
\begin{aligned}
& u(x, y)=\frac{\tau_{1}(x+y)+\tau_{1}(x-y)}{2}-\frac{1}{2} \int_{x+y}^{x-y} \nu_{1}(t) \mathrm{d} t \\
& +\frac{1}{4} \int_{x+y}^{x-y} \mathrm{~d} \eta \int_{x+y}^{\eta} \sum_{k=1}^{n} r_{k}(\eta) D_{-1 \eta}^{\beta_{k}} \tau_{2}(\eta) \mathrm{d} \xi
\end{aligned}
$$

of the Cauchy problem for the Eq. (1.1) in the domain of $\Delta_{2}$ with conditions

$$
u(x, 0)=\tau_{2}(x), x \in \overline{A_{2} B_{2}} ; \quad u_{y}(x, 0)=v_{2}(x), x \in A_{2} B_{2}
$$

and on the base of (1.8) we will obtain:

$$
\begin{aligned}
& u\left[\theta_{2}(x)\right]=\frac{\tau_{2}(-1)+\tau_{2}(x)}{2}-\frac{1}{2} \int_{-1}^{x} v_{2}(t) \mathrm{d} t+\frac{1}{4} \sum_{k=1}^{n} \int_{-1}^{x}(1+\eta) r_{k}(\eta) D_{-1 \eta}^{\beta_{k}} \tau_{2}(\eta) \mathrm{d} \eta \\
& \frac{1}{2} \tau_{2}^{\prime}(x)-\frac{1}{2} v_{2}(x)+\frac{x+1}{4} \sum_{k=1}^{n} r_{k}(x) D_{-1 x}^{\beta_{k}} \tau_{2}(x)=a_{2}(x) v_{2}(x)+b_{2}(x) \tau_{2}^{\prime}(x)+c_{2}(x) \tau_{2}(x)+d_{2}(x), \\
& \left(2 a_{2}(x)+1\right) v_{2}(x)=\left(1-2 b_{2}(x)\right) \tau_{2}^{\prime}(x)+\frac{x+1}{2} \sum_{k=1}^{n} r_{k}(x) D_{-1 x}^{\beta_{k}} \tau_{2}(x) \\
& -2 c_{2}(x) \tau_{2}(x)-2 d_{2}(x) .
\end{aligned}
$$

Theorem 2.1. If satisfies conditions

$$
\begin{aligned}
& a_{j}(x)+(-1)^{j}>0, \quad c_{j}(x) \geq 0, \quad\left(\frac{1-2 b_{j}(x)}{2 a_{j}(x)+(-1)^{j}}\right)^{\prime} \geq 0, \quad(j=1,2), \\
& p_{k}(q) \leq 0, r_{k}(q) \geq 0, \quad\left(\frac{(1-x) p_{k}(x)}{2 a_{1}(x)-1}\right)^{\prime} \leq 0,\left(\frac{(1+x) r_{k}(x)}{2 a_{2}(x)+1}\right)^{\prime} \geq 0, \quad(k=1,2, \ldots, n),
\end{aligned}
$$

then, the solution $u(x, y)$ of the Problem I is unique.

Proof. Known, that as, a function $u(x, y)$ is a solution of the Eq. (1.1) in the domain of $\Omega_{0}$, hence an identity:

$$
0 \equiv \iint_{\omega_{0}} u\left(u_{x x}+u_{y y}\right) \mathrm{d} x \mathrm{~d} y=\int_{-1}^{-q} u u_{y} \mathrm{~d} x+\int_{q}^{1} u u_{y} \mathrm{~d} x-\iint_{\Omega_{0}}\left(u_{x}^{2}+u_{x}^{2}\right) \mathrm{d} x \mathrm{~d} y
$$

i.e.

$$
\int_{-1}^{-q} \tau_{2}(x) v_{2}(x) \mathrm{d} x+\int_{q}^{1} \tau_{1}(x) v_{1}(x) \mathrm{d} x-\iint_{\Omega_{0}}\left(u_{x}^{2}+u_{y}^{2}\right) \mathrm{d} x \mathrm{~d} y \equiv 0
$$

is valid. Further, by virtue (2.3) and (2.5) from (2.8) at $d_{j}(x) \equiv 0$ we obtain:

$$
\int_{q}^{1} \frac{\left(1-2 b_{1}(x)\right) \tau_{1}^{\prime}(x) \tau_{1}(x)}{2 a_{1}(x)-1} \mathrm{~d} x+\int_{-1}^{-q} \frac{\left(1-2 b_{2}(x)\right) \tau^{\prime}{ }_{2}(x) \tau_{2}(x)}{2 a_{2}(x)+1} \mathrm{~d} x
$$




$$
\begin{aligned}
& -\sum_{k=1}^{n} \frac{1}{2 \Gamma\left(-\alpha_{k}\right)}\left[\int_{q}^{1} \frac{(1-x) p_{k}(x)}{2 a_{1}(x)-1} \mathrm{~d} x \int_{x}^{1} \frac{\tau_{1}(x) \tau_{1}(t)}{(t-x)^{1+\alpha_{k}}} \mathrm{~d} t-\int_{-1}^{-q} \frac{(1+x) r_{k}(x)}{2 a_{2}(x)+1} \mathrm{~d} x \int_{-1}^{x} \frac{\tau_{2}(x) \tau_{2}(t)}{(x-t)^{1+\alpha_{k}}} \mathrm{~d} t\right] \\
& -2 \int_{q}^{1} \frac{c_{1}(x) \tau_{1}^{2}(x)}{2 a_{1}(x)-1} \mathrm{~d} x-2 \int_{-1}^{-q} \frac{c_{2}(x) \tau_{2}^{2}(x)}{2 a_{2}(x)+1} \mathrm{~d} x-\iint_{\Omega_{0}}\left(u_{x}^{2}+u_{y}^{2}\right) \mathrm{d} x \mathrm{~d} y \equiv 0 .
\end{aligned}
$$

On a base to the formula [10]:

$$
|x-t|^{-\gamma}=\frac{1}{\Gamma(\gamma) \cos \frac{\pi \gamma}{2}} \int_{0}^{\infty} z^{\gamma-1} \cos [z(x-t)] \mathrm{d} z, \quad 0<\gamma<1,
$$

and

$$
\begin{aligned}
& -\sum_{k=1}^{n} \frac{\sin \left(\pi \alpha_{k}\right)}{2 \pi \cos \left(\pi\left(1+\alpha_{k}\right) / 2\right)} \int_{q}^{1} \frac{(1-x) p_{k}(x) \tau_{1}(x)}{2 a_{1}(x)-1} \mathrm{~d} x \int_{x}^{1} \tau_{1}(t) \mathrm{d} t \int_{0}^{\infty} z^{\alpha_{k}} \cos [z(t-x)] \mathrm{d} z \\
& +\sum_{k=1}^{n} \frac{\sin \left(\pi \beta_{k}\right)}{2 \pi \cos \left(\pi\left(1+\beta_{k}\right) / 2\right)} \int_{-1}^{-q} \frac{(1+x) r_{k}(x) \tau_{2}(x)}{2 a_{2}(x)+1} \mathrm{~d} x \int_{-1}^{x} \tau_{2}(t) \mathrm{d} t \int_{0}^{\infty} z^{\beta_{k}} \cos [z(x-t)] \mathrm{d} z \\
= & \sum_{k=1}^{n} \frac{\cos \left(\frac{\pi \alpha_{k}}{2}\right)}{\pi} \int_{q}^{1} \frac{(1-x) p_{k}(x) \tau_{1}(x)}{2 a_{1}(x)-1} \mathrm{~d} x \int_{x}^{1} \tau_{1}(t) \mathrm{d} t \int_{0}^{\infty} z^{\alpha_{k}}(\cos z t \operatorname{cox} z x+\sin z t \sin z x) \mathrm{d} z \\
& -\sum_{k=1}^{n} \frac{\cos \left(\frac{\pi \beta_{k}}{2}\right)}{\pi} \int_{-1}^{-q} \frac{(1+x) r_{k}(x) \tau_{2}(x)}{2 a_{2}(x)+1} \mathrm{~d} x \int_{-1}^{x} \tau_{2}(t) \mathrm{d} t \int_{0}^{\infty} z^{\beta_{k}}(\cos z t \operatorname{cox} z x+\sin z t \sin z x) \mathrm{d} z \\
= & \sum_{k=1}^{n} \frac{\cos \left(\frac{\pi \alpha_{k}}{2}\right)}{2 \pi} \int_{0}^{\infty} z^{\alpha_{k}} \mathrm{~d} z \int_{q}^{1} \frac{(x-1) p_{k}(x)}{2 a_{1}(x)-1} \mathrm{~d}\left[\left(\int_{x}^{1} \tau_{1}(t) \cos z t \mathrm{~d} t\right)^{2}+\left(\int_{x}^{1} \tau_{1}(t) \sin z t \mathrm{~d} t\right)^{2}\right] \\
& -\sum_{k=1}^{n} \frac{\cos \left(\frac{\pi \beta_{k}}{2}\right)}{2 \pi} \int_{0}^{\infty} z^{\beta_{k}} \mathrm{~d} z \int_{-1}^{-q} \frac{(1+x) r_{k}(x)}{2 a_{2}(x)+1} \mathrm{~d}\left[\left(\int_{-1}^{x} \tau_{2}(t) \cos z t \mathrm{~d} t\right)^{2}+\left(\int_{-1}^{x} \tau_{2}(t) \sin z t \mathrm{~d} t\right)^{2}\right] .
\end{aligned}
$$

Integrating integrals on $x$ by parts, obtain

$$
\begin{aligned}
& \sum_{k=1}^{n} \frac{(1-q) p_{k}(q) \cos \left(\frac{\pi \alpha_{k}}{2}\right)}{2 \pi\left(2 a_{1}(q)-1\right)} \int_{0}^{\infty} z^{\alpha_{k}}\left[\left(\int_{q}^{1} \tau_{1}(t) \cos z t \mathrm{~d} t\right)^{2}+\left(\int_{q}^{1} \tau_{1}(t) \sin z t \mathrm{~d} t\right)^{2}\right] \mathrm{d} z \\
& +\sum_{k=1}^{n} \frac{\cos \left(\frac{\pi \alpha_{k}}{2}\right)}{2 \pi} \int_{0}^{\infty} z^{\alpha_{k}} \mathrm{~d} z \int_{q}^{1} A^{\prime}{ }_{k}(x)\left[\left(\int_{x}^{1} \tau_{1}(t) \cos z t \mathrm{~d} t\right)^{2}+\left(\int_{x}^{1} \tau_{1}(t) \sin z t \mathrm{~d} t\right)^{2}\right] \mathrm{d} x \\
& -\sum_{k=1}^{n} \frac{(1-q) r_{k}(-q) \cos \left(\frac{\pi \beta_{k}}{2}\right)}{2 \pi\left(2 a_{2}(-q)+1\right)} \int_{0}^{\infty} z^{\beta_{k}}\left[\left(\int_{-1}^{-q} \tau_{2}(t) \cos z t \mathrm{~d} t\right)^{2}+\left(\int_{-1}^{-q} \tau_{2}(t) \sin z t \mathrm{~d} t\right)^{2}\right] \mathrm{d} z \\
& -\sum_{k=1}^{n} \frac{\cos \left(\frac{\pi \beta_{k}}{2}\right)}{2 \pi} \int_{0}^{\infty} z^{\beta_{k}} \mathrm{~d} z \int_{-1}^{-q} B^{\prime}{ }_{k}(x)\left[\left(\int_{-1}^{x} \tau_{2}(t) \cos z t \mathrm{~d} t\right)^{2}+\left(\int_{-1}^{x} \tau_{2}(t) \sin z t \mathrm{~d} t\right)^{2}\right] \mathrm{d} x .
\end{aligned}
$$


where $A_{k}(x)=\frac{(1-x) p_{k}(x)}{2 a_{1}(x)-1}, B_{k}(x)=\frac{(1+x) r_{k}(x)}{2 a_{2}(x)+1}$. Further, considering

$$
\begin{aligned}
& \int_{q}^{1} \frac{\left(1-2 b_{1}(x)\right) \tau_{1}^{\prime}(x) \tau_{1}(x)}{2 a_{1}(x)-1} \mathrm{~d} x+\int_{-1}^{-q} \frac{\left(1-2 b_{2}(x)\right) \tau_{2}^{\prime}(x) \tau_{2}(x)}{2 a_{2}(x)+1} \mathrm{~d} x \\
= & \frac{\left(1-2 b_{1}(1)\right) \tau_{1}^{2}(1)}{2\left(2 a_{1}(1)-1\right)}-\frac{\left(1-2 b_{1}(q)\right) \tau_{1}^{2}(q)}{2\left(2 a_{1}(q)-1\right)}-\frac{1}{2} \int_{q}^{1}\left(\frac{1-2 b_{1}(x)}{2 a_{1}(x)-1}\right)^{\prime} \tau_{1}^{2}(x) \mathrm{d} x \\
& \quad+\frac{\left(1-2 b_{2}(-q)\right) \tau_{2}^{2}(-q)}{2\left(2 a_{2}(-q)+1\right)}-\frac{\left(1-2 b_{2}(-1)\right) \tau_{2}^{2}(-1)}{2\left(2 a_{2}(-1)+1\right)}-\frac{1}{2} \int_{-1}^{-q}\left(\frac{1-2 b_{2}(x)}{2 a_{2}(x)+1}\right)^{\prime} \tau_{2}^{2}(x) \mathrm{d} x,
\end{aligned}
$$

and by virtue (2.11) from $(2.10)$ at $\varphi_{j}(x, y) \equiv 0(j=1,2)$ we will have:

$$
\begin{aligned}
& \int_{q}^{1}\left(\frac{1-2 b_{1}(x)}{2 a_{1}(x)-1}\right)^{\prime} \tau_{1}^{2}(x) \mathrm{d} x+\int_{-1}^{-q}\left(\frac{1-2 b_{2}(x)}{2 a_{2}(x)+1}\right)^{\prime} \tau_{2}^{2}(x) \mathrm{d} x+\int_{q}^{1} \frac{4 c_{1}(x) \tau_{1}^{2}(x)}{2 a_{1}(x)-1} \mathrm{~d} x \\
& +\int_{-1}^{-q} \frac{4 c_{2}(x) \tau_{2}^{2}(x)}{2 a_{2}(x)+1} \mathrm{~d} x \\
& -\sum_{k=1}^{n} \frac{(1-q) p_{k}(q) \cos \left(\frac{\pi \alpha_{k}}{2}\right)}{\pi\left(2 a_{1}(q)-1\right)} \int_{0}^{\infty} z^{\alpha_{k}}\left[\left(\int_{q}^{1} \tau_{1}(t) \cos z t \mathrm{~d} t\right)^{2}+\left(\int_{q}^{1} \tau_{1}(t) \sin z t \mathrm{~d} t\right)^{2}\right] \mathrm{d} z \\
& -\sum_{k=1}^{n} \frac{\cos \left(\frac{\pi \alpha_{k}}{2}\right)}{\pi} \int_{0}^{\infty} z^{\alpha_{k}} \mathrm{~d} z \int_{q}^{1} A^{\prime}{ }_{k}(x)\left[\left(\int_{x}^{1} \tau_{1}(t) \cos z t \mathrm{~d} t\right)^{2}+\left(\int_{x}^{1} \tau_{1}(t) \sin z t \mathrm{~d} t\right)^{2}\right] \mathrm{d} x \\
& +\sum_{k=1}^{n} \frac{(1-q) r_{k}(-q) \cos \left(\frac{\pi \beta_{k}}{2}\right)}{\pi\left(2 a_{2}(-q)+1\right)} \int_{0}^{\infty} z^{\beta_{k}}\left[\left(\int_{-1}^{-q} \tau_{2}(t) \cos z t \mathrm{~d} t\right)^{2}+\left(\int_{-1}^{-q} \tau_{2}(t) \sin z t \mathrm{~d} t\right)^{2}\right] \mathrm{d} z \\
& +\sum_{k=1}^{n} \frac{\cos \left(\frac{\pi \beta_{k}}{2}\right)}{\pi} \int_{0}^{\infty} z^{\beta_{k}} \mathrm{~d} z \int_{-1}^{-q} B^{\prime}{ }_{k}(x)\left[\left(\int_{-1}^{x} \tau_{2}(t) \cos z t \mathrm{~d} t\right)^{2}+\left(\int_{-1}^{x} \tau_{2}(t) \sin z t \mathrm{~d} t\right)^{2}\right] \mathrm{d} x \\
& +2 \iint_{\Omega_{0}}\left(u_{x}^{2}+u_{y}^{2}\right) \mathrm{d} x \mathrm{~d} y .
\end{aligned}
$$

Thus, taking (2.6) and (2.7) into account from (2.12) we can conclude, that $\tau_{1}(x) \equiv \tau_{2}(x) \equiv 0$ and $u_{x}(x, y) \equiv u_{y}(x, y) \equiv 0$ i.e. $u(x, y) \equiv$ const. Hence, based on $u(x, y) \in C\left(\bar{\Omega}_{0}\right)$ and boundary conditions $\left(4_{j}\right)$ at $\varphi_{j}(x, y) \equiv 0(j=1,2)$ we will get $u(x, y) \equiv 0$ in $\bar{\Omega}_{0}$ [12]. Further, on the bases of functional relations (2.3) and (2.5), taking into account $\tau_{1}(x) \equiv \tau_{2}(x) \equiv 0$ we will have $v_{1}(x) \equiv v_{2}(x) \equiv 0$, hence, the solution of the Cauchy problem for the Eq. (1.1) in the domains $\Delta_{j}$, identically equally to zero, i.e. $u(x, y) \equiv 0$ in the domains $\Delta_{j}$, as owing to uniqueness of solution of the Goursat problem, we will get that $u(x, y) \equiv 0$ in the domains $D_{j}(j=1,2,3)$. Thus, we have received, that $u(x, y) \equiv 0$ on the domain $\bar{\Omega}$ see [6-8]. 


\section{The existence of solution of the Problem I}

Theorem 3.1. If satisfies conditions (2.6), (2.7) and

$$
\begin{aligned}
& \varphi_{j}(x, y)=(x y)^{\gamma} \overline{\varphi_{j}}(x, y) ; \quad \overline{\varphi_{j}}(x, y) \in C\left(\overline{\sigma_{j}}\right), \quad 2<\gamma<3, \\
& g_{j}(x) \in C\left(\overline{I_{2+j}}\right) \cap C^{2}\left(I_{2+j}\right), \quad a_{j}(x), b_{j}(x), c_{j}(x), d_{j}(x) \in C\left(\overline{I_{j}}\right) \cap C^{2}\left(I_{j}\right) ; \\
& p_{k}(x) \in C\left(\overline{A_{1} B_{1}}\right) \cap C^{2}\left(A_{1} B_{1}\right), \quad q_{k}(x) \in C\left(\overline{A_{2} B_{2}}\right) \cap C^{2}\left(A_{2} B_{2}\right), \quad(k=\overline{1, n}),
\end{aligned}
$$

are fulfilled then the solution of the investigating problem is exist.

Proof. Considering (1.3) from (2.3), (2.5) and (2.6) we will respectively get:

$$
\begin{aligned}
\left(2 a_{1}(x)-1\right) v_{1}(x)= & \left(1-2 b_{1}(x)\right) \tau_{1}^{\prime}(x)+2 c_{1}(x) \int_{x}^{1} \tau^{\prime}{ }_{1}(t) \mathrm{d} t-2 c_{1}(x) \tau_{1}(1) \\
& -\frac{1-x}{2} \sum_{k=1}^{n} \frac{p_{k}(x)}{\Gamma\left(1-\alpha_{k}\right)}\left[(1-x)^{-\alpha_{k}} \tau_{1}(1)-\int_{x}^{1}(t-x)^{-\alpha_{k}} \tau^{\prime}{ }_{1}(t) \mathrm{d} t\right]-2 d_{1}(x) . \\
\left(2 a_{2}(x)+1\right) v_{2}(x)= & \left(1-2 b_{2}(x)\right) \tau_{2}^{\prime}(x)-2 c_{2}(x) \int_{-1}^{x} \tau^{\prime}{ }_{2}(t) \mathrm{d} t-2 c_{2}(x) \tau_{2}(-1) \\
& +\frac{x+1}{2} \sum_{k=1}^{n} \frac{r_{k}(x)}{\Gamma\left(1-\beta_{k}\right)}\left[(x+1)^{-\beta_{k}} \tau_{2}(-1)+\int_{-1}^{x}(x-t)^{-\beta_{k}} \tau^{\prime}{ }_{2}(t) \mathrm{d} t\right]-2 d_{2}(x) .
\end{aligned}
$$

Hence, on a base $u(x, y) \in C(\bar{\Omega})$ i.e. $\tau_{1}(1)=\varphi_{1}(1,0), \tau_{2}(-1)=\varphi_{1}(-1,0)$ we will receive

$$
\begin{aligned}
& \left(1-2 b_{1}(x)\right) \tau_{1}^{\prime}(x)+\int_{x}^{1}\left[\frac{1-x}{2} \sum_{k=1}^{n} \frac{p_{k}(x)(t-x)^{-\alpha_{k}}}{\Gamma\left(1-\alpha_{k}\right)}+2 c_{1}(x)\right] \tau^{\prime}{ }_{1}(t) \mathrm{d} t \\
= & \left(2 a_{1}(x)-1\right) v_{1}(x)+f_{21}(x), \\
& \left(1-2 b_{2}(x)\right) \tau_{2}^{\prime}(x)+\int_{-1}^{x}\left[\frac{x+1}{2} \sum_{k=1}^{n} \frac{r_{k}(x)(x-t)^{-\beta_{k}}}{\Gamma\left(1-\beta_{k}\right)}-2 c_{2}(x)\right] \tau^{\prime}{ }_{2}(t) \mathrm{d} t \\
= & \left(2 a_{2}(x)+1\right) v_{2}(x)+f_{22}(x),
\end{aligned}
$$

where

$$
\begin{aligned}
& f_{21}(x)=\frac{1}{2} \sum_{k=1}^{n} \frac{p_{k}(x)}{\Gamma\left(1-\alpha_{k}\right)}(1-x)^{1-\alpha_{k}} \varphi_{1}(1,0)+2 c_{1}(x) \varphi_{1}(1,0)+2 d_{1}(x), \\
& f_{22}(x)=2 d_{2}(x)-\frac{1}{2} \sum_{k=1}^{n} \frac{(x+1)^{1-\beta_{k}} r_{k}(x) \varphi_{1}(-1,0)}{\Gamma\left(1-\beta_{k}\right)}-2 c_{2}(x) \varphi_{1}(-1,0) .
\end{aligned}
$$

Now we should consider two cases : $b_{j}(x) \neq \frac{1}{2}$ and $b_{j}(x) \equiv \frac{1}{2}$ Let's $b_{j}(x) \neq \frac{1}{2}$, then from (3.4) and (3.5), we will accordingly get

$$
\tau_{1}^{\prime}(x)+\int_{x}^{1} K_{11}(x, t) \tau^{\prime}{ }_{1}(t) \mathrm{d} t=\tilde{f}_{21}(x),
$$




$$
\tau_{2}^{\prime}(x)+\int_{-1}^{x} K_{12}(x, t) \tau_{2}^{\prime}(t) \mathrm{d} t=\tilde{f}_{22}(x)
$$

where

$$
\begin{array}{ll}
K_{11}(x, t)=\frac{1}{1-2 b_{1}(x)}\left[\frac{1-x}{2} \sum_{k=1}^{n} \frac{p_{k}(x)(t-x)^{-\alpha_{k}}}{\Gamma\left(1-\alpha_{k}\right)}+2 c_{1}(x)\right], & q \leq x \leq t \leq 1 ; \\
K_{12}(x, t)=\frac{1}{1-2 b_{2}(x)}\left[\frac{1+x}{2} \sum_{k=1}^{n} \frac{r_{k}(x)(x-t)^{-\beta_{k}}}{\Gamma\left(1-\beta_{k}\right)}-2 c_{2}(x)\right], \quad-1 \leq t \leq x \leq-q ; \\
\tilde{f}_{2 j}(x)=\frac{1}{1-2 b_{j}(x)}\left[\left(2 a_{j}(x)+(-1)^{j}\right) v_{j}(x)+f_{2 j}(x)\right],
\end{array}
$$

at that, by virtue (3.2), (3.3) and owing to class of the function $u_{y}(x, 0)=v_{j}(x), x \in A_{j} B_{j}$ we will obtain

$$
\left|K_{1 j}(x, t)\right| \leq \text { const }, \quad\left|\tilde{f}_{2 j}(x)\right| \leq\left(x+(-1)^{j} q\right)^{-\varepsilon} \cdot \text { const }, \quad 0<\varepsilon<1, \quad j=1,2 .
$$

Notice, that the integral equations (3.6) and (3.7) are Volterra integral equations of second kind. Solving these equations by the method of consecutive approach it is found:

$$
\begin{aligned}
& \tau_{1}^{\prime}(x)=\frac{2 a_{1}(x)-1}{1-2 b_{1}(x)} v_{1}(x)+\int_{x}^{1} \frac{2 a_{1}(t)-1}{1-2 b_{1}(t)} \Re_{11}(x, t) v_{1}(t) \mathrm{d} t+F_{1}(x), \\
& \tau_{2}^{\prime}(x)=\frac{2 a_{2}(x)+1}{1-2 b_{2}(x)} v_{2}(x)+\int_{-1}^{x} \frac{2 a_{2}(t)+1}{1-2 b_{2}(t)} \Re_{12}(x, t) v_{2}(t) \mathrm{d} t+F_{2}(x),
\end{aligned}
$$

where $\Re_{1 j}(x, t)$ are resolvents of the kernels $K_{1 j}(x, t)$ and

$$
\begin{gathered}
F_{j}(x)=\frac{f_{2 j}(x)}{1-2 b_{j}(x)}-(j-1) \int_{-1}^{x} \frac{f_{22}(x)}{1-2 b_{2}(x)} \cdot \Re_{12}(x, t) \mathrm{d} t \\
+(j-2) \int_{x}^{1} \frac{f_{21}(x)}{1-2 b_{1}(x)} \cdot \Re_{11}(x, t) \mathrm{d} t,
\end{gathered}
$$

besides, on a base (3.8) we have $\left|\Re_{1 j}(x, t)\right| \leq$ const, hence, from (3.11) take into account (3.2) and (3.3) we will receive

$$
\left|F_{j}(x)\right| \leq \text { const, }(j=1,2) .
$$

Further, note that a solution of the problem $\mathrm{N}$ for the Eq. (1.1) in the domain of $\Omega_{0}$ with boundary conditions (1.5) and $u_{y}(x,+0)=v_{1}^{+}(x),(x, 0) \in A_{1} B_{1}, u_{y}(x,+0)=v_{2}^{+}(x)$, $(x, 0) \in A_{2} B_{2}$ is unique and represented on the form $[7,8]$ :

$$
u(x, y)=\int_{\sigma_{1}} \varphi_{1}(\xi, \eta) \frac{\partial}{\partial n} G(\xi, \eta ; x, y) \mathrm{d} S-\int_{\sigma_{2}} \varphi_{2}(\xi, \eta) \frac{\partial}{\partial n} G(\xi, \eta ; x, y) \mathrm{d} S
$$




$$
-\int_{q}^{1} v_{1}^{+}(t) G(t, 0 ; x, y) \mathrm{d} t+\int_{-1}^{-q} v_{2}^{+}(t) G(t, 0 ; x, y) \mathrm{d} t
$$

where $G(\xi, \eta ; x, y)$ is Greent function of the problem $\mathrm{N}$ for the equation $u_{x x}+u_{y y}=0$ in the domain $\Omega_{0}$ and it is represented as $[7,8]$ :

$$
G(\xi, \eta ; x, y)=\frac{1}{2 \pi} \ln \left|\frac{\theta_{1}\left(\frac{\ln v+\ln \bar{\mu}}{2 \pi i r}\right) \theta_{1}\left(\frac{\ln \bar{v}+\ln \bar{\mu}}{2 \pi i r}\right)}{\theta_{1}\left(\frac{\ln v-\ln \mu}{2 \pi i r}\right) \theta_{1}\left(\frac{\ln \bar{v}-\ln \mu}{2 \pi i r}\right)}\right|,
$$

where $v=\xi+i \eta, \bar{v}=\xi-i \eta, \mu=x+i y, \bar{\mu}=x-i y, r=\frac{1}{\pi i} \ln q, i^{2}=-1, \theta_{1}(\xi)=\theta_{1}\left(\xi \mid-\frac{1}{r}\right)$ is Theta function. From (3.13) at $y=0$, we will get a functional relation between $\tau_{1}^{+}(x)$ and $v_{1}^{+}(x)$ $\left(\tau_{2}^{+}(x)\right.$ and $\left.v_{2}^{+}(x)\right)$ on the piece $A_{1} B_{1}\left(A_{2} B_{2}\right)$ respectively, getting from the domain :

$$
\begin{gathered}
\tau_{1}^{+}(x)=\int_{\sigma_{1}} \varphi_{1}(\xi, \eta) \frac{\partial}{\partial n} G(\xi, \eta ; x, 0) \mathrm{d} S-\int_{\sigma_{2}} \varphi_{2}(\xi, \eta) \frac{\partial}{\partial n} G(\xi, \eta ; x, 0) \mathrm{d} S \\
-\int_{q}^{1} v_{1}^{+}(t) G(t, 0 ; x, 0) \mathrm{d} t+\int_{-1}^{-q} v_{2}^{+}(t) G(t, 0 ; x, 0) \mathrm{d} t, \\
\tau_{2}^{+}(x)=\int_{\sigma_{1}} \varphi_{1}(\xi, \eta) \frac{\partial}{\partial n} G(\xi, \eta ; x, 0) \mathrm{d} S-\int_{\sigma_{2}} \varphi_{2}(\xi, \eta) \frac{\partial}{\partial n} G(\xi, \eta ; x, 0) \mathrm{d} S \\
-\int_{q}^{1} v_{1}^{+}(t) G(t, 0 ; x, 0) \mathrm{d} t+\int_{-1}^{-q} v_{2}^{+}(t) G(t, 0 ; x, 0) \mathrm{d} t .
\end{gathered}
$$

Differentiating equalities (3.15) and (3.16) by $x$, obtain

$$
\tau_{j}^{\prime}(x)=\int_{-1}^{-q} v_{2}^{+}(t) \frac{\partial G(t, 0 ; x, 0)}{\partial x} \mathrm{~d} t-\int_{q}^{1} v_{1}^{+}(t) \frac{\partial G(t, 0 ; x, 0)}{\partial x} \mathrm{~d} t+\tilde{F}_{j}(x),
$$

where

$$
\tilde{F}_{j}(x)=\int_{\sigma_{1}} \varphi_{1}(\xi, \eta) \frac{\partial}{\partial x}\left[\frac{\partial}{\partial n} G(\xi, \eta ; x, 0)\right] \mathrm{d} S-\int_{\sigma_{2}} \varphi_{2}(\xi, \eta) \frac{\partial}{\partial x}\left[\frac{\partial}{\partial \eta} G(\xi, \eta ; x, 0)\right] \mathrm{d} S
$$

Having excluded $\tau_{j}^{\prime}(t),(j=1,2)$ from the relations (3.9), (3.10) and (3.17) we will get a system of integral equations:

$$
\left\{\begin{array}{l}
\frac{2 a_{1}(x)-1}{1-2 b_{1}(x)} \cdot v_{1}(x)+\int_{x}^{1} \frac{2 a_{1}(t)-1}{1-2 b_{1}(t)} \Re_{11}(x, t) v_{1}(t) \mathrm{d} t+\frac{1}{\pi} \int_{q}^{1} v_{1}(t) \tilde{K}_{2}(x, t) \mathrm{d} t \\
=\frac{1}{\pi} \int_{-1}^{-q} v_{2}(t) \tilde{K}_{2}(x, t) \mathrm{d} t+\tilde{F}_{1}(x)-F_{1}(x), \\
\frac{2 a_{2}(x)+1}{1-2 b_{2}(x)} v_{2}(x)+\int_{-1}^{x} \frac{2 a_{2}(t)+1}{1-2 b_{2}(t)} \Re_{12}(x, t) v_{2}(t) \mathrm{d} t-\frac{1}{\pi} \int_{-1}^{-q} v_{2}(t) \tilde{K}_{2}(x, t) \mathrm{d} t \\
=-\frac{1}{\pi} \int_{q}^{1} v_{1}(t) \tilde{K}_{2}(x, t) \mathrm{d} t+\tilde{F}_{2}(x)-F_{2}(x)
\end{array}\right.
$$


where

$$
\tilde{K}_{2}(x, t)=\frac{2 \ln |t|}{x \ln q}+\frac{1}{\pi}\left[\frac{1}{t-x}-\frac{t}{1-t x}+\sum_{n=1}^{\infty}\left(\frac{q^{2 n}}{t-q^{2 n} x}-\frac{q^{2 n} t}{1-q^{2 n} t x}-\frac{q^{-2 n} t}{1-q^{-2 n} t x}+\frac{q^{-2 n}}{t-q^{2 n} x}\right)\right] .
$$

Considering (2.6) the system (3.18) we will rewrite on the form:

$$
\left\{\begin{array}{l}
v_{1}(x)+\int_{q}^{1} K_{21}(x, t) v_{1}(t) \mathrm{d} t=\frac{1-2 b_{1}(x)}{\pi\left(2 a_{1}(x)-1\right)} \cdot \int_{-1}^{-q} v_{2}(t) \tilde{K}_{2}(x, t) \mathrm{d} t+F_{1}^{*}(x), \\
v_{2}(x)+\int_{-1}^{-q} K_{22}(x, t) v_{2}(t) \mathrm{d} t=-\frac{1-2 b_{2}(x)}{\pi\left(2 a_{2}(x)+1\right)} \int_{q}^{1} v_{1}(t) \tilde{K}_{2}(x, t) \mathrm{d} t+F_{2}^{*}(x)
\end{array}\right.
$$

where

$$
\begin{aligned}
& K_{21}(x, t)=\left\{\begin{array}{l}
\frac{2 a_{1}(t)-1}{1-2 b_{1}(t)} \cdot \frac{1-2 b_{1}(x)}{2 a_{1}(x)-1} \cdot \Re_{11}(x, t)+\frac{1-2 b_{1}(x)}{2 a_{1}(x)-1} \cdot \tilde{K}_{2}(x, t), \quad x \leq t \leq 1, \\
\frac{1-2 b_{1}(x)}{2 a_{1}(x)-1} \cdot \tilde{K}_{2}(x, t), \quad 0 \leq t \leq x,
\end{array}\right. \\
& F_{1}^{*}(x)=\frac{1-2 b_{1}(x)}{2 a_{1}(x)-1} \cdot\left(\tilde{F}_{1}(x)-F_{1}(x)\right), \quad F_{2}^{*}(x)=\frac{1-2 b_{1}(x)}{2 a_{1}(x)+1} \cdot\left(\tilde{F}_{2}(x)-F_{2}(x)\right) .
\end{aligned}
$$

Notice that the functions $\tilde{F}_{j}(x)$ was investigated in the research works $[7,8]$ and for this functions takes place $\left|\tilde{F}_{j}(x)\right| \leq \operatorname{const}\left(x+(-1)^{j} q\right)^{\gamma-3}(2<\gamma<3)$. Hence, taking into account (2.7), (3.1)-(3.3) and (3.12) from (3.19), we will conclude that $F_{1}^{*}(x) \in C^{2}(q, 1)$ $\left(F_{2}^{*}(x) \in C^{2}(-1,-q)\right)$ and $F_{j}^{*}(x)$ can tend to infinity an order of less one at $x \rightarrow(-1)^{j-1} q$, and at $x \rightarrow(-1)^{j-1}(j=1,2)$ it is limited.

Thus, the system of integral equation (3.18) reduce to the Fredholm integral equations of the second kind, by the known method of the Karleman-Vekua [13], just as in works [7, 8]. Note, that the unique solvability of the Fredholm integral equations of the second kind follows from the uniqueness of solution of the Problem I and from the theory integral equations. Solving the system of integral equations (3.18), we will found $v_{1}(x)$ and $v_{2}(x)$ [7], further owing to account (3.9), (3.10) and $\tau_{1}(q)=\varphi_{2}(q, 0), \tau_{2}(-q)=\varphi_{2}(-q, 0)$ from (3.15) and (3.16) we will find $\tau_{j}(x),(j=1,2)$.

Hence, after founding $\tau_{1}(x)$ and $v_{1}(x), \tau_{2}(x)$ and $v_{2}(x)$, the solution of the Problem I can be restored in the domain $\Omega_{0}$ as the solution of the Problem $\mathbf{N}$ (3.13), and in domains $\Delta_{j}(j=1,2)$ as the solution of the Cauchy problem. The solution of the Problem I in domains of $D_{j}(\mathrm{j}=1,2)$, we can restore as a solution of the Goursat problem with conditions (1.6) and, where $h_{j}(x)(j=1,2)$ are traces of solution of the Cauchy problems in domains $\Delta_{j}$ $(j=1,2)$, on the line $y-(-1)^{j} x=q$, and reciprocally in domain $D_{3}$ as the solution of the Goursat problem with conditions $(j=1,2)$ where $\tilde{h}_{j}(t)(j=1,2)$ are traces of solution of the Goursat problems in domains $D_{j}(j=1,2)$.

The theorem is proved on the case of $b_{j}(x) \neq \frac{1}{2}$. 
Let's $b_{j}(x)=\frac{1}{2}$, then from (2.3) and $(2.5$,$) we will accordingly get$

$$
\begin{aligned}
& 2 c_{1}(x) \tau_{1}(x)+\frac{1-x}{2} \int_{x}^{1} \sum_{k=1}^{n} \frac{p_{k}(x)}{\Gamma\left(-\alpha_{k}\right)}(t-x)^{-\alpha_{k}-1} \tau_{1}(t) \mathrm{d} t=\left(1-2 a_{1}(x)\right) v_{1}(x)-2 d_{1}(x), \\
& 2 c_{2}(x) \tau_{2}(x)-\frac{1+x}{2} \int_{-1}^{x} \sum_{k=1}^{n} \frac{r_{k}(x)}{\Gamma\left(-\beta_{k}\right)}(x-t)^{-\beta_{k}-1} \tau_{2}(t) \mathrm{d} t=-\left(1+2 a_{2}(x)\right) v_{2}(x)-2 d_{2}(x) .
\end{aligned}
$$

Note that the integral equations (3.20) and (3.21) at $c_{j}(x) \neq 0$ are Volterra integral equations of second kind.

Solving these equations by the method of consecutive approach it is found:

$$
\begin{aligned}
& \tau_{1}(x)=\frac{1-2 a_{1}(x)}{2 c_{1}(x)} \cdot v_{1}(x)+\int_{x}^{1} \frac{1-2 a_{1}(t)}{2 c_{1}(t)} \tilde{\Re}_{11}(x, t) v_{1}(t) \mathrm{d} t+M_{1}(x), \\
& \tau_{2}(x)=-\frac{2 a_{2}(x)+1}{2 c_{2}(x)} v_{2}(x)-\int_{-1}^{x} \frac{2 a_{2}(t)+1}{2 c_{2}(t)} \tilde{\Re}_{12}(x, t) v_{2}(t) \mathrm{d} t+M_{2}(x),
\end{aligned}
$$

where $\tilde{\Re}_{1 j}(x, t)$ are resolvents of kernel $\tilde{K}_{1 j}(x, t)$ of the integral equations (3.20) and (3.21), where

$$
\begin{array}{ll}
\tilde{K}_{11}(x, t)=\frac{1-x}{4 c_{1}(x)} \sum_{k=1}^{n} \frac{p_{k}(x)(t-x)^{-\alpha_{k}-1}}{\Gamma\left(-\alpha_{k}\right)}, & q \leq x \leq t \leq 1, \\
\tilde{K}_{12}(x, t)=-\frac{1+x}{4 c_{2}(x)} \sum_{k=1}^{n} \frac{r_{k}(x)(x-t)^{-\beta_{k}-1}}{\Gamma\left(-\beta_{k}\right)}, & -1 \leq t \leq x \leq-q \\
M_{1}(x)=-\int_{x}^{1} \frac{d_{1}(t)}{c_{1}(t)} \tilde{\Re}_{11}(x, t) \mathrm{d} t-\frac{d_{1}(x)}{c_{1}(x)}, & M_{2}(x)=-\int_{-1}^{x} \frac{d_{2}(t)}{c_{2}(t)} \tilde{\Re}_{12}(x, t) \mathrm{d} t-\frac{d_{2}(x)}{c_{2}(x)},
\end{array}
$$

besides, $\left|\tilde{K}_{1 j}(x, t)\right| \leq|x-t|^{-\varepsilon}$ const, $0<\varepsilon<1 ;\left|M_{j}(x)\right| \leq$ const, $(j=1,2)$.

Further, this problem can be investigated similarly for the case of $b_{j}(x) \neq \frac{1}{2}$.

Now we will assume, that $c_{j}(x)=0$ then the integral equations (3.20) and (3.21) are Volterra integral equations of the first kind. Known that such equations can be solved leading them to the Volterra integral equations of the second kind. As a final result in this case we can formulate the following Lemma:

Lemma 3.1. If satisfies conditions (2.7), (3.1)-(3.3) and

$$
2 a_{j}(1)+(-1)^{j}>0, \quad d_{j}\left(-(-1)^{j}\right)=0, \quad c_{j}(x) \equiv 0, \quad 1-2 b_{j}(x) \equiv 0, \quad(j=1,2),
$$

then the solution of the investigating problem is exist. 


\section{References}

[1] Nakhushev A. M., The loaded equations and their applications. M. Nauka, (2012). 232.

[2] Eleev V. A., About some boundary value problems for mixed type loaded equations of the second and third equations. Diff. Equat., 30 (2) (1994), 230-237.

[3] Kaziev V., On a Darboux problem for the one loaded integral-differential equations of the second order. Diff. Equat., 14 (1) (1978), 181-184.

[4] Lanin I. N., Boundary value problems for the loaded hyperbolic-parabolic type equations of the third equations. Diff. Equat., 17 (1) (1981), 97-106.

[5] Sabitov K. B., Initial-Boundary Problem for Parabolic-Hyperbolic Equation with Loaded Summand. Izvestiya Vysshikh Uchebnykh Zavedenii. Matematika 6 (2015), 23-33.

[6] Abdullaev O. Kh., Non-local boundary value problem for the mixed type equations on the third order in double-connected domains. Journal of P. D. E., 27 (4) (2014), 283-292.

[7] Abdullaev O. Kh., Boundary value problem for a loaded equation elliptic-hyperbolic type in double connected domain. Journal Collection of Scientific Works of KRASEC, 1 (8) (2014).

[8] Abdullaev O. Kh., About a method of research of the non-local problem for the loaded mixed type equation in double-connected domain. Bulletin KRASEC. Phys. Math. Sci, 9 (2) (2014), 11-16.

[9] Abdullaev O. Kh., Non-local problem for the loaded mixed type equation with the integrated operators. International conference. Differential equations and mathematical modeling Ulan-Ude, 2015. 21-23.

[10] Smirnov M. M., Mixed Type Equations, M. Nauka., 2000.

[11] Agmon S. Nirenberg L. Protter M. H., A maximum principle for a class of hyperbolic equation and application to equations elliptic-hyperbolic type. Communs Pure Appl. Math., 1 (1953), 455-470.

[12] Bitsadze A.V., Differential Equations of Mixed Type. Mac. Millan. Co., New York. (1964).

[13] Muskheleshvili N. I., Singularity integral equations. M: Nauka. (1968) 512. 